\title{
Índice neutrófilos/linfocitos: un predictor de mortalidad en paciente con infección por SARS-CoV-2
}

\author{
The neutrophiles/lymphocytes index: is a predictor of mortality in a patient with infection by SARS-CoV-2 \\ $O$ indice de neutrófilos/linfócitos um preditor de mortalidade em um paciente com infecção por SARS-CoV-2
}

Michell Montalvo Aguilar, ${ }^{*}$ César Augusto González López*

\section{RESUMEN}

Introducción: En diciembre de 2019 se dio a conocer que en la ciudad de Wuhan (China) se había presentado una nueva serie de casos de neumonía, al parecer originadas por un nuevo tipo de coronavirus. El 07 de enero de 2020 el nuevo coronavirus fue anunciado oficialmente por las autoridades chinas como el agente causal de dichas infecciones. El índice neutrófilos/linfocitos (INL) ha tomado interés como biomarcador sérico para definir la gravedad. Cataudella y colaboradores reportaron la relación proporcional del INL con la gravedad de la neumonía y algunos desenlaces adversos; además, se ha observado que puede ser predictor de mortalidad en unidades de cuidados intensivos.

Material y métodos: Se realizó un estudio prospectivo, longitudinal, analítico, se ingresaron a todos los pacientes con diagnósticos de infección por SARSCoV-2 en un periodo de tiempo de abril a agosto de 2020, en la Unidad de Cuidados Intensivos del Hospital Regional «Lic. Adolfo López Mateos».

Resultados: La población estudiada se compuso de 59 sujetos; no obstante, para el análisis se excluyeron a dos pacientes por datos insuficientes. De los 57 restantes, $61.4 \%(n=35)$ fueron hombres y $38.6 \%(n=22)$ mujeres. Con respecto a la curva de ROC, el cual es un análisis cuya finalidad determina un valor de corte con alta sensibilidad a predecir un suceso (en nuestro objetivo mortalidad), arrojó un resultado significativo sólo en el índice neutrófilos/linfocitos cuando el paciente se encontraba en la $\mathrm{UCl}$ con un área bajo la curva de 0.728 (IC 0.597-0.860) ( $p=0.004)$; mostró una especificidad de $80 \%$ cuando el índice tenía un valor de 10.35 o mayor en estos pacientes. El cálculo para este parámetro no fue significativo cuando el paciente ingresó a urgencias y la hipótesis se negó con un área bajo la curva de 0.628 (IC 0.480-0.777).

Conclusiones: Entre nuestros resultados destaca que el valor reportado promedio en los pacientes que fallecieron durante su estancia en la UCl fue de 54.56 \pm 10.89 años versus aquellos vivos que fue de $48.23 \pm 11.42$ ( $p=0.037$ ), lo que concuerda con lo reportado en la literatura por diversos autores. Se decidió buscar algún punto de corte en la población mexicana, donde Reyes-Gálvez y colegas reportaron en sepsis que un valor de 18.1 a 36 o incluso mayor es un valor del índice neutrófilos/linfocitos que se asocia con un estado severo o crítico de la enfermedad y se relaciona de forma modesta con la escala de APACHE. De acuerdo con estos puntos de corte, la población que se analizó no reportó asociaciones significativas mientras que se encontraban en los servicios de urgencias, posiblemente a que la respuesta inflamatoria aguda aún no se desarrollaba en su totalidad o la sintomatología primaria sólo es insuficiencia respiratoria que ameritaba su hospitalización y posterior pase a la $\mathrm{UCl}$ donde este valor fue significativo $(p=0.022)$. Palabras clave: Índice, neutrófilo/linfocito, SARS-CoV-2, predictor, mortalidad, Unidad de Cuidados Intensivos.

\section{ABSTRACT}

Introduction: In December 2019 it was announced that in the city of Wuhan (China) a new series of pneumonia cases had occurred, apparently caused by a new type of coronavirus. On January 07, 2020, the new coronavirus was officially announced by the Chinese authorities as the causative agent of these infections. The neutrophil/lymphocyte ratio (INL) has taken on interest as a serum biomarker to define severity. Cataudella et al, reported the proportional relationship of INL with the severity of pneumonia and some adverse outcomes, in addition, it has been observed that it can be a predictor of mortality in Intensive Care Units. Material and methods: A prospective, longitudinal, analytical study was carried out in which all patients with diagnoses of SARS-CoV-2 infection were admitted in a period of time from April-August 2020, in the intensive care unit of the Regional Hospital «Lic. Adolfo López Mateos».

\section{* Hospital Regional «Lic. Adolfo López Mateos», ISSSTE. \\ Recibido: 09/10/2020. Aceptado: 24/10/2020.}

Citar como: Montalvo AM, González LCA. Índice neutrófilos/linfocitos: un predictor de mortalidad en paciente con infección por SARS-CoV-2. Med Crit. 2021;35(3):130-135. https://dx.doi.org/10.35366/100001

www.medigraphic.com/medicinacritica
Results: The study population consisted of 59 subjects, however, for the analysis, two patients were excluded due to insufficient data. Of the remaining $57,61.4 \%(n=35)$ were men and $38.6 \%(n=22)$ were women. Regarding the ROC curve, which is an analysis whose purpose determines a cut-off value with high sensitivity to predict an event (in our mortality objective), a significant result was carried out and produced only in the neutrophil/lymphocyte index when the patient was in the ICU with an area under the curve of $0.728(\mathrm{Cl}$ 0.597-0.860) $(\mathrm{p}$ $=0.004$ ), it showed a specificity of $80 \%$ when the index had a value of 10.35 or greater in these patients. The calculation for this parameter in while the patient was admitted to the emergency room was not significant and the hypothesis was denied with an area under the curve of 0.628 ( $\mathrm{Cl}$ 0.480-0.777).

Conclusions: Among our results, it stands out that the average reported value in patients who died during their stay in the ICU was $54.56 \pm 10.89$ years vs those alive, which was $48.23 \pm 11.42(p=0.037)$, which is consistent with that reported in the literature by various authors. it was decided to look for a cut-off point in the Mexican population, where Reyes-Gálvez et al. reported in sepsis that a value of 18.1 to 36 or even higher is a value of the neutrophil/lymphocyte index that is associated with a severe or critical state of the disease and is modestly associated with the APACHE scale. According to these cut-off points, the population analyzed did not report significant associations while they were in the emergency services, possibly because the acute inflammatory response had not yet been fully developed, or the primary symptomatology is only respiratory failure that merited his hospitalization and subsequent transfer to the ICU where this value was significant $(p=0.022)$.

Keywords: Index, neutrophile/lymphocyte, SARS-CoV-2, predictive, mortality, Intensive Care Unit.

\section{RESUMO}

Introdução: Em dezembro de 2019 foi anunciado que na cidade de Wuhan (China) havia ocorrido uma nova série de casos de pneumonia, aparentemente causados por um novo tipo de coronavírus. Em 7 de janeiro de 2020, o novo coronavírus foi anunciado oficialmente pelas autoridades chinesas como o agente causador dessas infecções. A razão neutrófilos/linfócitos (INL) tem se tornado um biomarcador sérico para definir a gravidade. Cataudella et al, relataram a relação proporcional do INL com a gravidade da pneumonia e alguns desfechos adversos, além disso, observou-se que pode ser um preditor de mortalidade em unidades de terapia intensiva.

Material e métodos: Foi realizado um estudo prospectivo, longitudinal e analítico no qual todos os pacientes com diagnóstico de infecção por SARSCoV-2 foram internados no período de abril de 2020 a agosto de 2020, na Unidade de Terapia Intensiva do Hospital Regional "Lic. Adolfo López Mateos". Resultados: População do estudo consistiu de 59 indivíduos, entretanto, 2 pacientes foram excluídos para análise devido a dados insuficientes. Dos 57 restantes, $61.4 \%(n=35)$ eram homens e 38.6\% $(n=22)$ eram mulheres. Em relação à curva $R O C$, que é uma análise cujo objetivo determina um valor de corte com alta sensibilidade para predizer um evento (em nosso objetivo de mortalidade), um resultado significativo foi realizado e produzido apenas no índice de neutrófilos/linfócitos quando o paciente estava na UTI com área sob a curva de 0.728 (IC: 0.597-0.860) ( $p=0.004$ ), apresentou especificidade de $80 \%$ quando o índice teve valor igual ou superior a 10.35 nesses pacientes. $O$ cálculo desse parâmetro durante a internação do paciente no pronto-socorro não foi significativo e a hipótese foi negada com área sob a curva de 0.628 (IC: 0.480-0.777).

Conclusões: Dentre os nossos resultados, destaca-se que o valor médio relatado em pacientes que morreram durante a internação na UTI foi de 54.56 \pm 10.89 anos vs vivos, que foi de $48.23 \pm 11.42(p=0.037)$, o que está de acordo com o relatado na literatura de vários autores. Optou-se por buscar um ponto de corte na população mexicana, onde Reyes-Gálvez et al. relataram na sepse que um valor de 18.1 a 36 ou até mais alto é um valor do índice de neutrófilos/linfócitos que está associado a um estado grave ou crítico da doença e está modestamente associado à escala APACHE. De acordo com esses pontos de corte, a população analisada não relatou associações significativas enquanto estiveram nos serviços de emergência, possivelmente porque a resposta inflamatória aguda ainda não havia se desenvolvido totalmente, ou a sintomatologia primária era apenas insuficiência respiratória que necessitava 
hospitalização e posterior transferência para a UTI onde este valor foi significativo $(p=0.022)$.

Palavras-chave: Índice, neutrófilo/linfócito, SARS-CoV-2, preditivo, mortalidade, Unidade de Terapia Intensiva.

\section{INTRODUCCIÓN}

En diciembre de 2019 se dio a conocer que en la ciudad de Wuhan (China) se había presentado una nueva serie de casos de neumonía, al parecer originadas por un nuevo tipo de coronavirus. El 07 de enero de 2020 el nuevo coronavirus fue anunciado oficialmente por las autoridades chinas como el agente causal de dichas infecciones. ${ }^{1}$

EI SARS-CoV-2 es un betacoronavirus, de ARN de sentido positivo, no segmentado; subgénero Sarbecovirus, subfamilia Orthocoronavirinae. Es una enfermedad infecciosa respiratoria aguda, emergente, que se propaga a través del tracto respiratorio. ${ }^{2}$

En algunas series de casos que se publicaron en China la incidencia de enfermedad grave con requerimientos de ingreso a las unidades de cuidados intensivos se encuentra alrededor de $6.1 \%$ para la totalidad de los infectados y de $26 \%$ para los que se encuentran en las áreas de hospitalización. ${ }^{3}$

Los marcadores bioquímicos frecuentemente utilizados para evaluar a este tipo de pacientes son: perfil renal, estado ácido-base, glucosa, dímero D, fibrinógeno, troponina ultrasensible, ferritina, proteína $\mathrm{C}$ reactiva, ácido láctico, procalcitonina, entre otros. Sin embargo, uno que aún no se ha mencionado y que es de suma importancia como predictor de mortalidad es la relación neutrófilos/ linfocitos. ${ }^{4}$

El índice neutrófilos/linfocitos (INL) ha tomado interés como biomarcador sérico para definir la gravedad. Cataudella y colaboradores reportaron la relación proporcional del INL con la gravedad de la neumonía y algunos desenlaces adversos; además, se ha observado que puede ser predictor de mortalidad en unidades de cuidados intensivos. ${ }^{5,6}$

La inflamación juega un papel importante en la fisiopatología de COVID-19, como la proporción de neutrófilos/ linfocitos, los cuales reflejan de forma indirecta el estado inflamatorio del paciente. ${ }^{7}$ El INL se calcula como el recuento absoluto de neutrófilos dividido entre el recuento absoluto de linfocitos. ${ }^{8}$ En los últimos años ha sido validado como marcador pronóstico en diversos trastornos. ${ }^{4,9}$

En la actualidad, pocos estudios han evaluado el papel del INL en relación con la gravedad de COVID-19.4,10

En un estudio prospectivo realizado en el Hospital Ditan de Beijing, por Liu y colegas, se evidenció en 61 pacientes con infección por SARS-CoV-2 la disminución del recuento de linfocitos relacionada con el progreso de la enfermedad. ${ }^{11}$ Por lo anterior, se demostró que el INL era un factor significativo que afectaba la incidencia de enfermedad grave y tenía un valor predictivo de mortalidad. ${ }^{12}$
Caos y su equipo han informado una alta incidencia de linfopenia en pacientes con COVID-19, haciendo que la relación neutrófilos/linfocitos se haya consolidado como posible indicador de pronóstico a corto plazo en pacientes ingresados a la unidad de cuidados intensivos. ${ }^{13}$

Un estudio indicó que grandes cantidades de citocinas proinflamatorias en suero se asociaron con inflamación y daño pulmonar extenso en COVID-19, similar al síndrome respiratorio agudo severo (SARS) y la infección por coronavirus. ${ }^{14}$ Un número exponencial de estudios ha encontrado que una proporción elevada de neutrófilos a linfocitos (NLR), que refleja, al menos parcialmente, la respuesta inflamatoria, resultó ser un factor pronóstico independiente de resultados adversos. ${ }^{15}$

Aproximadamente de 20 al $30 \%$ de los casos desarrollarán una enfermedad grave y algunos necesitarán una mayor intervención, la cual se otorgará en la Unidad de Cuidados Intensivos. ${ }^{15}$ El síndrome de dificultad respiratoria aguda, el choque, la lesión cardiaca aguda, la lesión renal aguda y la disfunción orgánica se pueden presentar de manera inminente en pacientes con diagnóstico de infección por SARS-CoV-2 una vez ingresados en la Unidad de Cuidados Intensivos, lo que conduciría un resultado clínico deficiente para el paciente. ${ }^{16}$

\section{MATERIAL Y MÉTODOS}

Se realizó un estudio prospectivo, longitudinal y analítico en el cual se ingresaron a todos los pacientes con diagnóstico de infección por SARS-CoV-2 con prueba confirmada por PCR; en un periodo de tiempo comprendido entre abril y agosto de 2020, que ingresaron a la Unidad de Cuidados Intensivos del Hospital Regional «Lic. Adolfo López Mateos». De los cuales se extrajeron datos como el índice de neutrófilos, linfocitos y su índice tanto al ingreso al Servicio de Urgencias como al Servicio de Terapia Intensiva. Se plasmaron los datos en una tabla del programa Excel, así como se registraron también los fallecimientos de los pacientes y el índice que se presentó.

\section{RESULTADOS}

La población estudiada se compuso de 59 sujetos; no obstante, para el análisis se excluyeron a dos pacientes por datos insuficientes. De los 57 restantes, $61.4 \%(n=$ 35) fueron hombres y $38.6 \%(n=22)$ mujeres.

Al analizar los datos descriptivos se observó una tendencia en prevalencia de sujetos mayores a 50 años $56.1 \%(n=32)$, lo cual se ha considerado en la literatura como un factor de riesgo para la mortalidad de los pacientes con SARS-CoV-2. En tanto, la mortalidad final durante el seguimiento del estudio fue de $47.4 \%$ (n = 27) (Tabla 1); la mayoría de estos pacientes fue del sexo masculino con $60 \%(n=21)(p=0.016)$. 
De acuerdo a la mortalidad, se ha teorizado sobre el índice neutrófilos/linfocitos, el cual se ha asociado con la gravedad de la enfermedad, los puntos de corte para la población mexicana de acuerdo con nuestro estudio, a su ingreso a urgencias se concentró la mayor parte en riesgo bajo y moderado con un porcentaje de $38.6 \%$ $(n=22)$ en cada diagnóstico; mientras que cuando los pacientes trascendieron a la Unidad de Cuidados Intensivos el mayor porcentaje se concentró en riesgo alto 0 grave con $43.6 \%(n=24)$.

En relación a los datos biométricos, todos se comportaron como no paramétricos, por lo tanto, se expresaron en medianas y rangos. El número de linfocitos en urgencias fue de $0.9419(0.29-2.55)$, en la UCI la mediana reportó ligero decremento con 0.8993 (0.176.55). El valor de los neutrófilos en urgencias fue de 10.03 (1.38-31.14) y en la UCI de 10.81 (2.30-44.89) y, por último, el valor del índice de neutrófilos/linfocitos en urgencias fue de 13.87 (1.39-75.95) y en la UCI de 19.53 (0.81-85.15) (Tabla 1).

Los análisis inferenciales para describir la relación de la mortalidad con las diversas variables del estudio demandaron pruebas como t de Student, $\chi^{2}$ y curvas de ROC. En relación a la primera de las pruebas se observó que cuando los pacientes ingresaban al Servicio de Urgencias no mostraron diferencias significativas al compararse los egresos vivos con los fallecidos $(p=0.577$ ) (Figura 1); mientras que una vez que el paciente se encontraba en la
UCl el panorama cambiaba drásticamente y estos datos mostraban una significancia estadística $(p=0.006)$.

Con el fin de describir la relación que presentó el índice neutrófilos/linfocitos en torno a la mortalidad en la población de estudio, se calculó la media poblacional de éste a su ingreso al Servicio de Urgencias y en su estancia en la $\mathrm{UCl}$ y en ninguno de los casos reportó una significancia estadística ( $p=0.278$ y 0.075$)$. Al agregar el punto de corte de edad $\geq 50$ años en las mismas instancias los resultados fueron semejantes al anterior ( $p=$ 0.289 y 0.215 ), por lo cual se asumió que tanto la edad como un valor arbitrario del índice de neutrófilos/linfocitos de acuerdo a la población son datos independientes en relación a la mortalidad en la población de estudio, por lo que se procedió a realizar una curva de ROC, con el fin de calcular un valor sensible a mortalidad en la población.

Con respecto a la curva de ROC, el cual es un análisis cuya finalidad determina un valor de corte con alta sensibilidad a predecir un suceso (en nuestro objetivo mortalidad), arrojó un resultado significativo sólo en el índice neutrófilos/linfocitos cuando el paciente se encontraba en la $\mathrm{UCl}$ con un área bajo la curva de 0.728 (IC 0.597-0.860) $(p=0.004)$, mostró una especificidad de $80 \%$ cuando el índice tenía un valor de 10.35 o mayor en estos pacientes.

El cálculo para este parámetro no fue significativo cuando el paciente ingresó a urgencias y la hipótesis se negó con un área bajo la curva de 0.628 (IC 0.480-0.777).

Tabla 1: Datos descriptivos de la población en estudio con diagnóstico de infección por SARS-CoV-2 en un periodo de tiempo de abril a agosto de 2020 en la Unidad de Cuidados Intensivos del Hospital Regional «Lic. Adolfo López Mateos».

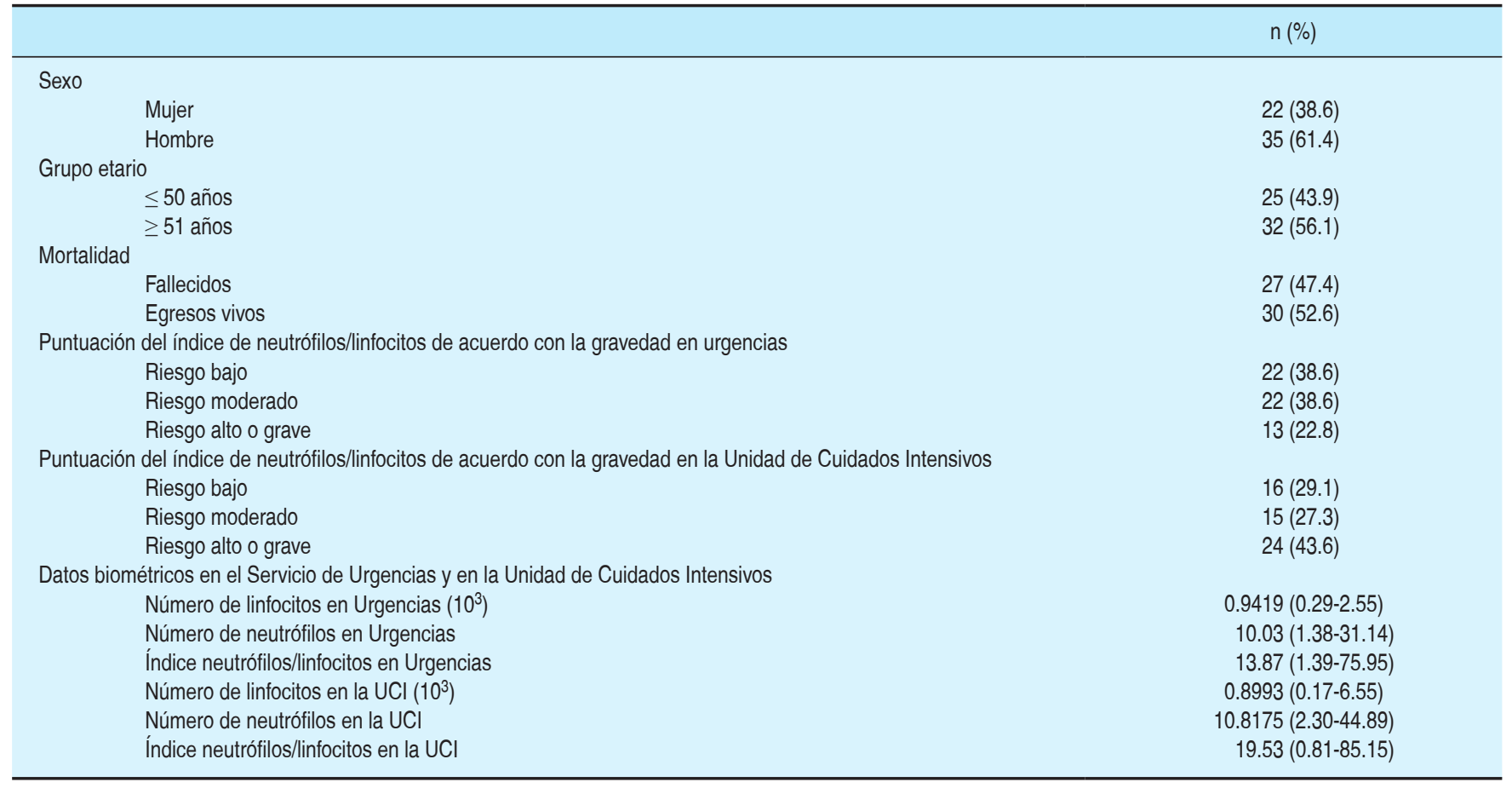

$\mathrm{UCl}=$ Unidad de Cuidados Intensivos. 


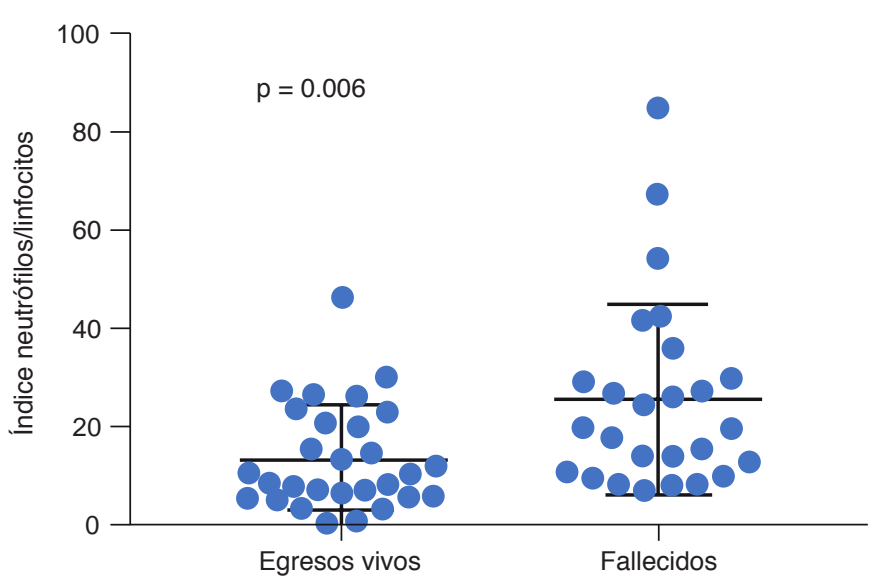

Figura 1: Análisis de asociación de acuerdo con el desenlace en la población estudio, y al índice de neutrófilos/linfocitos en la Unidad de Cuidados Intensivos.

\section{DISCUSIÓN}

Desde diciembre de 2019 se reportaron los primeros casos de SARS-CoV-2 en el mundo, el sistema de salud chino, al igual que el mexicano, se vio desafiado al tener un promedio de 2,000 a 4,000 casos nuevos por día y con un incremento de la mortalidad en pacientes con comorbilidades o de edad mayor a 50 años.

Los coronavirus han existido desde tiempos inmemorables y uno de los síntomas más habituales era el resfriado común, ${ }^{17,18}$ a diferencia de ello los pacientes con SARS-CoV-2 tienen un patrón respiratorio y presenta un tropismo a órganos diana con receptores ACE2. ${ }^{3} \mathrm{EI}$ virus se une a estos receptores que se expresan de forma dominante en pulmones, tracto gastrointestinal, renal y cardiaco, lo que explica la sintomatología presente en los pacientes infectados. ${ }^{19}$

Mientras el virus ingresa en las células, el hospedero desarrolla una respuesta inmunológica importante que activa el complejo principal de histocompatibilidad, y en casos graves se han reportado niveles altos de citocinas proinflamatorias (IL-2, IL-7, IL-10, MCP1, TNF- $\alpha$, TFG- $\beta$, INF- $\gamma$, quimiocinas y otras sustancias más). No obstante, en el sector público es prácticamente imposible evaluarlo a pesar de que se recomiende calcular en casos críticos de UCl dímero D, IL-6 o procalcitonina, por lo que nuestro principal objetivo fue evaluar el índice neutrófilos/linfocitos, que ha sido asociado a la gravedad de la enfermedad con valores superiores a 3.1 en combinación con la edad de los pacientes mayores a 50 años.

Entre nuestros resultados destaca que el valor reportado promedio en los pacientes que fallecieron durante su estancia en la $U C l$ fue de $54.56 \pm 10.89$ años versus aquellos vivos que fue de $48.23 \pm 11.42(p=0.037)$ (Figura 2), lo que concuerda con lo reportado en la li- teratura por diversos autores. Aunado a la edad, se ha reportado que estos pacientes sufren de leucopenia y linfocitopenia que al conjugarse con otros problemas en los que el virus afecta a otros órganos los desenlaces son peores. ${ }^{8}$ Desafortunadamente, por la naturaleza del protocolo y la saturación hospitalaria que se vivió, se tuvieron datos perdidos por causas externas al grupo de investigación, por lo que se decidió basar el análisis sólo en el índice neutrófilos/linfocitos, debido a que la mayoría de los pacientes tenían reportes de biometría hemática en su estancia en la UCl.

Por lo anterior, se decidió buscar algún punto de corte en la población mexicana, Reyes-Gálvez y colaboradores reportaron en sepsis que un valor de 18.1 a 36 o incluso mayor es un valor del índice neutrófilos/linfocitos que se asocia con un estado severo o crítico de la enfermedad y se asocia de forma modesta con la escala de APACHE. De acuerdo con estos puntos de corte, la población que se analizó no reportó asociaciones significativas mientras se encontraban en los servicios de urgencias, posiblemente a que la respuesta inflamatoria aguda aún no se desarrollaba en su totalidad o la sintomatología primaria sólo es insuficiencia respiratoria que ameritaba su hospitalización y posterior pase a la $\mathrm{UCI}$ donde este valor fue significativo ( $p=0.022)$.

La relación que encontró Reyes-Gálvez y colegas podría replicarse en nuestro estudio; sin embargo, se debe replicar la investigación y posiblemente recomendar realizar escalas como APACHE o incluso agregar algún otro indicador. Por lo anterior, en nuestro grupo se decidió aplicar un análisis para determinar una sen-

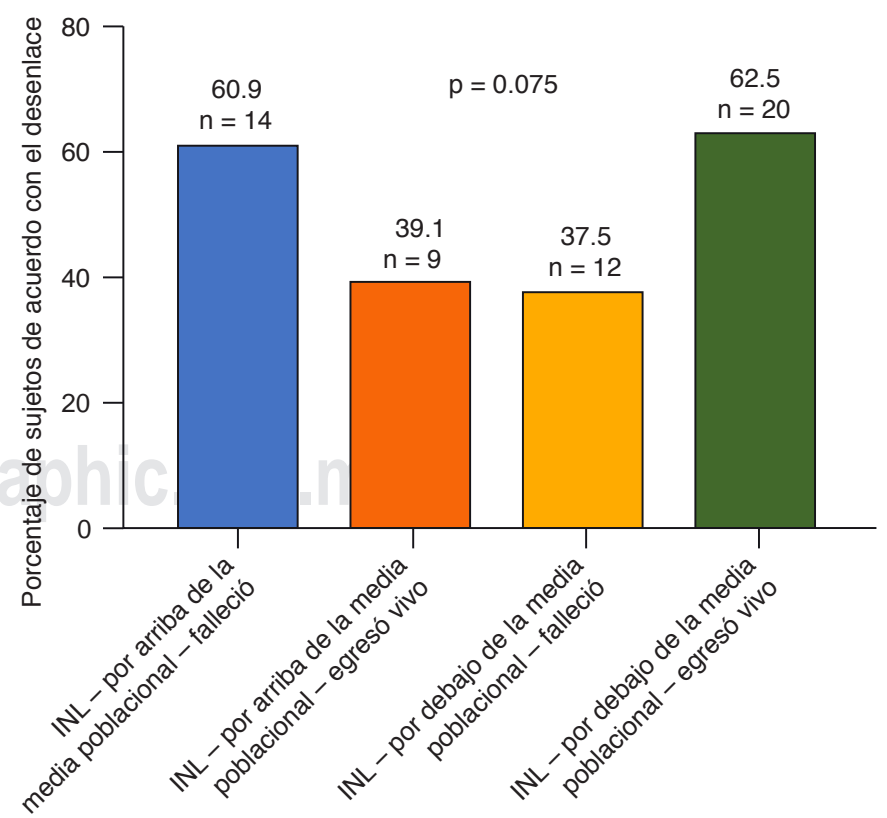

Figura 2: Porcentaje de sujetos con índice de neutrófilos/linfocitos en la Unidad de Cuidados Intensivos con un desenlace de mortalidad. 


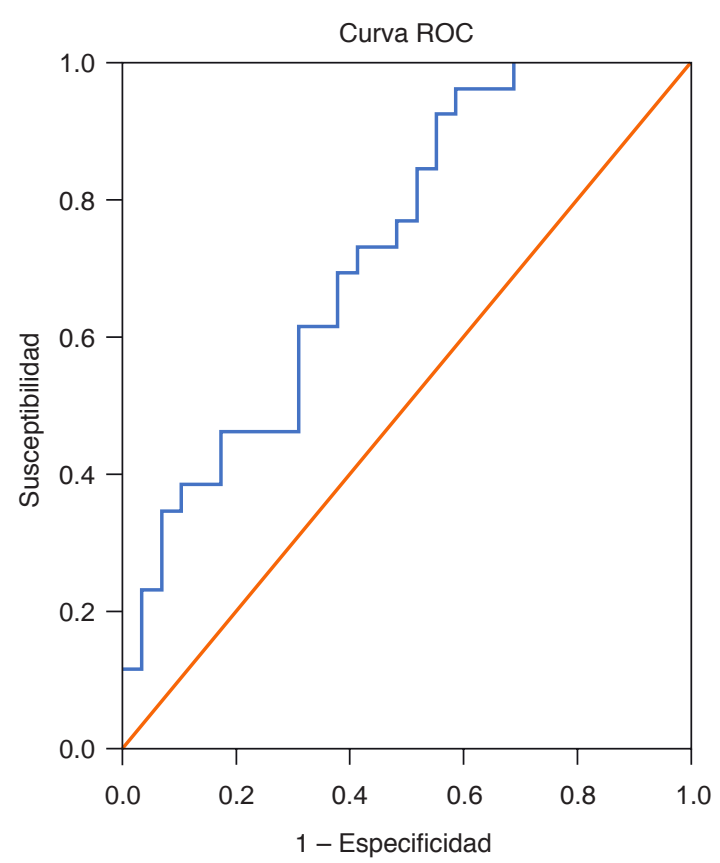

Área bajo la curva

Variables resultado de contraste: índice neutrófilos/ linfocitos UCI.

\begin{tabular}{ccccc} 
& & & \multicolumn{2}{c}{ Intervalo de confianza asintótico al 95\% } \\
\cline { 4 - 5 } Área & Error típ. $^{*}$ & Sig. asintótica & Límite inferior & Límite superior \\
\hline 0.728 & 0.067 & 0.004 & 0.597 & 0.860 \\
\hline
\end{tabular}

* Bajo el supuesto no paramétrico. ${ }^{\ddagger}$ Hipótesis nula: área verdadera $=0.5$

Figura 3: Curva ROC para evaluar susceptibilidad y especificidad al índice neutrófilos/linfocitos en pacientes con SARS-CoV-2 en la Unidad de Cuidados Intensivos.

sibilidad predictiva de un desenlace fatídico a través de una curva de ROC.

En los datos significativos anteriormente mostrados en la Figura 3, la curva demostró poseer un valor de 0.8 cuando el índice neutrófilos/linfocitos en la $\mathrm{UCl}$ era mayor a 10.35; los valores de edad, neutrófilos, linfocitos y el índice neutrófilos/linfocitos en urgencias fueron factores independientes, por lo que se debe estar al tanto de la evolución del paciente. Los datos anteriores muestran que en nuestra población la infección por SARSCoV-2 no tiene un patrón o comportamiento específico $y$, que al igual que en la literatura, se limitan a hablar de mecanismos asociados a los receptores ACE2 sin tener un panorama certero aún.

En nuestra población reconocemos las limitantes en torno a la no inclusión de otras variables; sin embargo, se detectó un estudio de Che-Morales, en el que se analizaron neumonías adquiridas en la comunidad y se tomó como punto de corte un índice $\leq 7.2,{ }^{10}$ que predecía los desenlaces graves u hospitalizados, esto podría ser muestra de que nuestros resultados de la curva de ROC se asemejan al mostrar una tendencia de incremento cuando el valor sobrepasa las 8.4 unidades que reportó Reyes-Gálvez.

Por último, reconocemos que los alcances de nuestro estudio no fueron los idóneos, por lo que sugerimos que en posteriores estudios se tomen en cuenta algunos parámetros que se han asociado con la mortalidad a fin de hacer un mayor alcance y tener conclusiones con mayor robustez.

Como el caso de Feng-Pang y su grupo, quienes hablan de valores como saturación parcial de oxígeno, PCR, procalcitonina y lactato deshidrogenasa aunado a una edad de 50 años, ${ }^{11}$ a una escala como APACHE y el índice neutrófilos/linfocitos. No obstante, reconocemos nuestras fortalezas y sabemos que hasta ahora es el único reporte que existe en torno a SARS-CoV-2 en población mexicana que evalúa el índice neutrófilos/linfocitos.

\section{CONCLUSIONES}

1. La edad promedio de los pacientes que fallecieron fue de $54.56 \pm 10.89$ vs $48.23 \pm 11.42(p=0.037)$.

2. El área bajo la curva del índice neutrófilos/linfocitos fue de 0.728 (IC 0.597-0.860) y se detectó que un valor de 10.35 muestra $80 \%$ de sensibilidad ante un evento fatídico.

3. Los valores de neutrófilos/linfocitos tanto en urgencias como en la $\mathrm{UCI}$ no se asociaron de forma significativa; no así, el valor del índice neutrófilos/linfocitos reportó una $\mathrm{p}=0.006$ en la $\mathrm{UCl}$.

4. Los valores o puntos de corte de pacientes con sepsis respecto al índice neutrófilos/linfocitos predijo de forma significativa la mortalidad del estudio.

\section{REFERENCIAS}

1. Luis B, Martin I, Ignacio M, María C, Javier P. Índice neutrófilolinfocito como factor pronóstico de covid-19. Medicina (Buenos Aires). 2020;80(Supl. III):31-36.

2. José $\mathrm{C}$, Arturo $\mathrm{C}$. Indice neutrófilo/linfocito como biomarcador sérico asociado con neumonía adquirida en comunidad. Rev Med Inst Mex Seguro Soc. 2018;56(6):537-543.

3. José S, María S, Juan G. Predictores se mortalidad en pacientes con COVID-19. Archivos de Medicina. 2020;16(26):1-3.

4. Reyes-Gálvez JA, Gracida-Mancilla NI, Enríquez- Santos D, Carrillo-Esper R. Índice neutrófilos-linfocitos como predictor de gravedad y mortalidad en pacientes con sepsis abdominal. Med Int Méx. 2016;32(1):41-47.

5. Jiangshan L, Ciliang J, Shaorui $H$, Xiaoli Z, Meifang $Y, X i ~ J$, et al. High neutrophil-to-lymphocyte ratio associated with progression to critical illness in older patients with COVID-19: a multicenter retrospective study. Aging. 2020;12(14):13849-13859.

6. Roujian L, Xiang Z, Juan L. Genomic characterisation and epidemiology of 2019 novel coronavirus: implications for virus origins and receptor binding. Lancet. 2020;395:565-574.

7. Guo YR, Cao QD, Hong ZS, Tan YY, Chen SD, Jin HJ, et al. The origin, transmission and clinical therapies on coronavirus disease 2019 (COVID-19) outbreak-an update on the status. Military Med Res. 2020;7(1):11. Available from: http://dx.doi.org/10.1186/ s40779-020-00240-0.

8. Palacios CM, Santos E, Velázquez CMA, León JM. COVID-19, una emergencia de salud pública mundial. Revista Clínica 
Española. 2021;221(1):55-61. Available from: http://dx.doi. org/10.1016/j.rce.2020.03.001.

9. Socorro FS, Fernandes Jr PC, Barbosa SMJ, Lima VC, Fontes $\mathrm{W}$, Freitas-Junior $\mathrm{R}$, et al. The neutrophil-to-lymphocyte ratio: a narrative review. Ecancer. 2016;10. Available from: http://dx.doi. org/10.3332/ecancer.2016.702.

10. Zhang B, Zhou X, Zhu C, Song Y, Feng F, Qiu Y, et al. Immune phenotyping based on the neutrophil-to-lymphocyte ratio and igg level predicts disease severity and outcome for patients with COVID-19. Front Mol Biosci. 2020;7. Available from: http://dx.doi. org/10.3389/fmolb.2020.00157.

11. Chan AS, Rout A. Use of neutrophil-to-lymphocyte and platelet-to-lymphocyte ratios in COVID-19. J Clin Med Res. 2020;12(7):448-453. Available from: http://dx.doi.org/10.14740/ jocmr4240.

12. Fu J, Kong J, Wang W, Wu M, Yao L, Wang Z, et al. The clinical implication of dynamic neutrophil to lymphocyte ratio and D-dimer in COVID-19: a retrospective study in Suzhou China. Thrombosis Research. 2020;192:3-8. Available from: http:// dx.doi.org/10.1016/j.thromres.2020.05.006.

13. Yang A-P, Liu J, Tao W, Li H. The diagnostic and predictive role of NLR, d-NLR and PLR in COVID-19 patients. Int Immunopharmacol. 2020;84:106504. Available from: http:// dx.doi.org/10.1016/j.intimp.2020.106504.

14. Liu Y, Du X, Chen J, Jin Y, Peng L, Wang HHX, et al. Neutrophilto-lymphocyte ratio as an independent risk factor for mortality in hospitalized patients with COVID-19. Journal of Infection. 2020;81(1):e6-12. Available from: http://dx.doi.org/10.1016/j. jinf.2020.04.002.

15. Devaux CA, Rolain J-M, Colson P, Raoult D. New insights on the antiviral effects of chloroquine against coronavirus: what to expect for COVID-19? International Journal of Antimicrobial
Agents. 2020;55(5):105938. Available from: http://dx.doi. org/10.1016/j.ijantimicag.2020.105938.

16. Pan F, Yang L, Li Y, Liang B, Li L, Ye T, et al. Factors associated with death outcome in patients with severe coronavirus disease-19 (COVID-19): a case-control study. Int J Med Sci. 2020;17(9):1281-1292. Available from: http://dx.doi.org/10.7150/ ijms.46614.

17. Lippi G, Wong J, Henry BM. Hypertension and its severity or mortality in Coronavirus Disease 2019 (COVID-19): a pooled analysis. Polish Archives of Internal Medicine. 2020;130(4):304309. Available from: http://dx.doi.org/10.20452/pamw.15272.

18. Martínez-Urbistondo D, Beltrán A, Beloqui O, Huerta A. El índice neutrófilo/linfocito como marcador de disfunción sistémica endotelial en sujetos asintomáticos. Nefrología. 2016;36(4):397-403. Available from: http://dx.doi.org/10.1016/j. nefro.2015.10.018.

19. Liu J, Liu Y, Xiang P, Pu L, Xiong H, Li C, et al. Neutrophilto-lymphocyte ratio predicts critical illness patients with 2019 coronavirus disease in the early stage. J Trans/ Med. 2020;18(1). Available from: http://dx.doi.org/10.1186/s12967-020-02374-0.

\section{Patrocinios: Ninguno. \\ Relación de conflicto de intereses: Ninguno.}

Correspondencia:

Michell Estrella Montalvo Aguilar

Avenida Universidad Núm. 1321,

Col. Florida, 01030.

E-mail: dramichellmontalvo@gmail.com 